\title{
Aparato Experimental de Escolha: Desenvolvimento de Sistema Informatizado para Estudo de Comportamento de Escolha
}

\author{
Giovana Escobal ${ }^{1}$ \\ Celso Goyos \\ Laboratório de Aprendizagem Humana, Multimídia Interativa e Ensino Informatizado \\ da Universidade Federal de São Carlos, São Carlos, São Paulo, Brasil
}

\begin{abstract}
Resumo
Realizar escolha é fundamental para a conduta adaptativa de qualquer indivíduo em seu ambiente social, principalmente para pessoas com deficiência intelectual ou atraso no desenvolvimento que não possuem essas habilidades em seu repertório. Conduzir estudos sobre escolha que exigem a apresentação de estímulos de difícil manipulação podem ter sua viabilidade prejudicada devido aos procedimentos de apresentação serem complicados para se executar. O objetivo deste estudo foi o desenvolvimento de um Aparato Experimental de Escolha capaz de mecanizar a disponibilização e controle de cada exposição de tarefas de trabalho, o que representa uma vantagem uma vez que as tarefas podem ser preparadas rápida e antecipadamente e apresentadas diversas vezes, o que proporcionaria diminuição do tempo de aplicação, além de possibilitar o registro fidedigno das respostas, da latência, do tempo de execução da tarefa e de liberação da consequência. $\mathrm{O}$ aparato consiste de uma mesa redonda giratória com botões acoplados e permite a aplicação de esquemas de reforçamento de razão fixa e variável, intervalo fixo e variável, além do uso de esquemas concorrentes simples ou concorrentes com encadeamento. O controle é feito diretamente pelo computador, que armazena os dados automaticamente e diminui as possibilidades de erros manuais. A utilização de recursos mecânicos aliados aos computacionais parece bastante promissora, com a possibilidade adicional de proporcionar um uso mais frequente e por um maior número de pessoas.
\end{abstract}

Palavras-chave: Aparato experimental, escolha, preferência, tecnologia informatizada, registro fidedigno.

\section{Experimental Apparatus of Choice: Development of Computerized System for the Study of Choice Behavior}

\section{Abstract}

Perform choice is fundamental to adaptive behavior of any individual in his/her social environment, especially for intellectually or developmentally delayed people that often lack these skills in their re-

Endereço para correspondência: Laboratório de Aprendizagem Humana, Multimídia Interativa e Ensino Informatizado, Departamento de Psicologia, Universidade Federal de São Carlos, Via Washington Luís, km 235, Caixa Postal 676, São Carlos, SP, Brasil 13565-905. E-mail: giovanaescobal@hotmail.com e celsogoyos2@ gmail.com

Os autores agradecem à Fundação de Amparo à Pesquisa do Estado de São Paulo (FAPESP) pelo Auxílio à Pesquisa que tornou possível o desenvolvimento do aparato experimental de escolha (Processo número 2007/04958-3) e por bolsa de pós-doutorado à primeira autora (Processo número 2010/11201-9) e ao Conselho Nacional de Desenvolvimento Científico e Tecnológico $(\mathrm{CNPq})$ pela bolsa de produtividade à pesquisa ao segundo autor (Processo número 306921/2010-3). Adicionalmente, os autores agradecem a Empresa Insight pela competência e parceria no desenvolvimento do Aparato Experimental de escolha. 
pertoire. Conducting studies of choice that require the presentation of difficult-to-handle items may face insurmountable barriers due to choice procedures that are complicated to implement. The objective of the present study was to develop an Experimental Apparatus of Choice capable of mechanize the delivery and control of each exposure, which is an advantage since the tasks can be set up quickly and previously and presented several times, which would allow the reduction of application time, and enable reliable record of responses, latency time of task execution and consequence presentation. The apparatus consists of a rotating roundtable with buttons attached. The apparatus allows the application of reinforcement schedules of fixed and variable ratios, and fixed and variable intervals, besides the use of simple concurrent schedules of reinforcement or concurrent-chain schedules of reinforcement. The control is done directly by the computer, which automatically stores data and decreases the chances of manual errors. The use of computing resources seems quite promising, with the additional possibility of providing more frequent use to a greater number of people. A validation of the experimental apparatus of choice has been made by a study.

Keywords: Experimental apparatus, choice, preference, computerized technology, reliable record.

\section{Aparato Experimental de Elección: Desarrollo de Sistema Informatizado para el Estudio del Comportamiento de Elección}

\section{Resumen}

Efectuar elección es fundamental para el comportamiento adaptativo de cualquier individuo en su entorno social, especialmente para las personas con discapacidad intelectual o retraso en el desarrollo, a menudo carecen de estas habilidades en su repertorio. Conducir estudios de elección que requieren la presentación de artículos de difíciles manipulación, pueden tener su viabilidad afectada debido a los procedimientos de presentación que son difíciles de realizar. El objetivo de este estudio fue desarrollar un Aparato Experimental de elección capaz de mecanizar la exposición y control de cada exposición, que es una ventaja ya que las tareas se pueden construir rápidamente y por adelantado y presentado varias veces, lo cual proporcionaría tiempo de aplicación reducido, además de permitir el registro fidedigno de las respuestas, el tiempo de latencia de la ejecución de la tarea y la liberación de refuerzo. El aparato consta de una mesa redonda giratoria con botones adjuntos. El aparato permite la aplicación de esquemas de refuerzo de proporción fijo y variable, intervalo fijo y variable, además del uso de esquemas competidores simples o competidores con encadenamiento. El control se realiza directamente por el ordenador, que almacena automáticamente los datos y reduce las posibilidades de errores manuales. El uso de los recursos informáticos parece bastante prometedora, con la posibilidad adicional de proporcionar un uso más frecuente y un mayor número de personas. Una validación del aparato experimental de elección ha sido hecha por un estudio.

Palabras clave: Aparato experimental, elección, preferencia, tecnología computarizada, registro fidedigno.

Realizar escolha é fundamental para a conduta adaptativa de qualquer indivíduo em seu ambiente social. Escolher está na base dos comportamentos considerados importantes na definição atual de deficiência intelectual, proposta pela Associação Americana de Retardo Mental de 1992 e 2002, no que tange aos grandes grupos de habilidades sociais, práticas e conceituais
(Luckasson et al., 2002; Luckasson et al., 1992).

Os benefícios de se oferecer oportunidades de escolha a pessoas com necessidades educacionais especiais, sobretudo àquelas com déficit pronunciado de conduta nos grupos de habilidades sociais, práticas e conceituais são amplos. A todo momento, os organismos fazem escolhas e demonstram preferências. Desde as situações 
mais simples (e.g., o que comer em uma refeição), até as mais complexas, (e.g., qual carreira profissional seguir, com quem se casar, ter ou não filhos), o comportamento de escolha é exigido. Escolher está na base do processo de tomada de decisões, assim como na base do processo de resolução de problemas, e está no cerne de questões relativas ao controle e autocontrole, à autonomia, à dignidade, à autodeterminação e à cidadania do indivíduo (Dunlap et al., 1994; Hanna \& Ribeiro, 2005).

A importância de um repertório comportamental que envolva escolha é mais evidentemente sentida quando a pessoa encontra-se privada da oportunidade de realizá-las, ou por falta de condições facilitadoras, ou por falta de repertório próprio, ou por ambas. Por isso, o ensino do comportamento de escolher deve constituir-se objetivo fundamental nos programas educativos para pessoas com deficiência intelectual severa ou profunda (Escobal \& Goyos, 2008; Fisher, Thompson, Piazza, Crosland, \& Gotjen, 1997).

A fim de implementar com sucesso intervenções baseadas em reforçamento em pesquisas básicas, translacionais e aplicadas, e em programas de ensino, é fundamental realizar avaliações de preferência para identificar estímulos reforçadores efetivos para uso com participantes ou pacientes (Piazza, Fisher, Bowman, \& Blakeley-Smith, 1999).

Uma forma de avaliar preferência envolve a disponibilização de repetidas oportunidades de escolha entre duas ou mais alternativas, simultaneamente disponíveis, para determinar qual é escolhida com mais frequência. Estímulos reforçadores potenciais podem ser identificados de diversas maneiras, e uma hierarquia de estímulos reforçadores potenciais pode ser construída por meio de métodos indiretos, tais como entrevistas e observações informais ou métodos diretos, em geral, mais precisos que os métodos indiretos, tais como Avaliação de Preferência com Estímulo Único (Pace, Ivancic, Edwards, Iwata, \& Page, 1985), Avaliação de Preferência de Escolha Pareada (Fisher et al., 1992), Avaliação de Preferência com Múltiplos Estímulos (Carr, Nicolson, \& Higbee, 2000; DeLeon \& Iwata, 1996) e Avaliação de Preferência com Operante Livre
(Roane, Vollmer, Ringdahl, \& Marcus, 1998).

De maneira geral, as pesquisas sobre a avaliação de preferência têm empregado estímulos tangíveis e facilmente manipuláveis, tais como: comestíveis, brinquedos, materiais escolares, e outros (Carr et al., 2000; DeLeon \& Iwata, 1996; Fisher et al., 1992; Pace et al., 1985; Roane et al., 1998).

Alguns autores têm sugerido que o uso de figuras impressas não gera uma hierarquia de preferência confiável (Hanley, Iwata, \& Lindberg, 1999; Higbee, Car, \& Harrison, 1999), principalmente quando a resposta de selecionar uma figura não resulta no acesso ao item correspondente.

Hanley et al. (1999) examinaram as preferências de quarto indivíduos com atraso de desenvolvimento através da apresentação de figuras que representavam várias atividades. Inicialmente, as escolhas não mostraram nenhuma preferência clara. Na condição subsequente, escolher uma figura resultou em um breve acesso à atividade representada. Quando as escolhas passaram a produzir consequências diferenciais (acesso à atividade), a preferência emergiu claramente.

Higbee et al. (1999) conduziram um estudo para determinar a possibilidade de usar figuras em avaliações de preferência. Avaliações de preferência com múltiplos estímulos foram conduzidas com dois adultos diagnosticados com deficiência intelectual usando itens tangíveis e cartões com figuras impressas representando os mesmos estímulos. Os resultados indicaram que o método com itens tangíveis previu os efeitos reforçadores dos estímulos com maior acurácia que a avaliação com figuras. Há várias possíveis razões pelas quais a avaliação com figuras falhou em determinar reforçadores eficazes acuradamente. Embora os participantes pudessem identificar (tato), oralmente ou através de gestos, os estímulos nos cartões, eles não respondiam (mando) às figuras da mesma maneira que respondiam aos itens tangíveis, talvez pela inexperiência dos participantes em usar figuras para requisitar um item tangível. Outra razão para que os participantes respondessem de forma diferente para os cartões poderia ser a diferença de procedimento entre as duas avaliações. 
$\mathrm{Na}$ avaliação com itens tangíveis, cada vez que o participante selecionava um item, ele ganhava 20 s de acesso a esse item. Na avaliação com os cartões, entretanto, a resposta de seleção não resultava no acesso ao item correspondente. Se o acesso ao estímulo for concebido com o reforçador que mantém a resposta de seleção, então, não fornecer acesso ao item seria similar a operação de extinção.

Resultados diferentes foram encontrados por Groskreutz e Graff (2009) que compararam três formatos diferentes de avaliação: avaliação tangível com o acesso ao estímulo, avaliação com figura com o acesso ao estímulo e avaliação com figura sem acesso ao estímulo. Avaliações de reforçadores foram conduzidas para verificar os efeitos reforçadores. Na Fase 1, os autores examinaram os formatos de avaliação em blocos alternados de tentativas. Na Fase 2, examinaram os resultados da condição de avaliação com figura sem acesso ao estímulo administrada primeiro, seguida pelos outros formatos de avaliação. Os autores pré-testaram habilidades de emparelhamento entre cada figura e o seu estímulo tangível correspondente, o que pode ser uma habilidade necessária ao substituir estímulos tangíveis por figuras. Na Fase 1, Groskreutz e Graff (2009) indicaram os mesmos estímulos mais preferidos para 4 de 5 participantes, independente do formato da avaliação. Na Fase 2, os resultados das avaliações de reforçadores indicaram que o formato de avaliação com figura sem acesso ao estímulo pode ser acurado para alguns participantes, mas não para todos quando as avaliações são concluídas em sua totalidade. Os resultados também indicaram que entre esses três formatos, o formato com figuras sem acesso ao estímulo foi o administrado em menor tempo. Os autores mencionaram que avaliações com figuras sem acesso ao estímulo poderiam ser feitas com o auxílio de um computador, que poderia ser mais eficiente em termos de tempo e esforço dos aplicadores, pois o programa é apropriadamente e especificamente desenvolvido para apresentar os estímulos e registrar as respostas de escolha. Então, apresentar figuras digitais através de um programa de computador seria uma extensão do formato de avaliação com figuras impressas apresentados sobre uma mesa.

Conyers et al. (2002) realizaram um estudo em que nove indivíduos com deficiência intelectual foram submetidos a procedimentos de escolha forçada em três condições (estímulos tangíveis, figuras digitalizadas e o nome falado desses mesmos estímulos), utilizando um delineamento de reversão. A avaliação foi realizada com estímulos comestíveis, e depois repetida com estímulos variados não comestíveis. Os resultados demonstraram que alguns participantes selecionaram seus estímulos preferidos de maneira consistente tanto com cada figura ou estímulo tangível. Os autores sugerem ainda que para a generalidade dos resultados desse estudo, outros estímulos poderiam ser testados em avaliações de preferência.

Se, por um lado, os procedimentos com estímulos tangíveis mostram eficiência (Fisher et al., 1992; Pace et al., 1985), por outro lado a aplicação de procedimentos deste tipo implica em preparação e controle manual de cada oportunidade de escolha, por meio da seleção e apresentação dos estímulos durante o teste e registro de dados, que incluí registrar a quantidade de vezes que um determinado estímulo foi escolhido e/ ou o período de tempo que durou a interação ou o consumo; demandando frequentemente a presença de assistentes para auxiliar no andamento das atividades. Pode-se dizer, assim, que essa forma de avaliação de preferência com estímulos tangíveis, demanda bastante tempo e uma série de comportamentos por parte do pesquisador, que concorrem com as próprias tarefas da coleta de dados da pesquisa. O presente estudo sugere uma nova maneira de realizar avaliações de preferência, através de um Aparato Experimental de Escolha. Os procedimentos de escolha poderão ser realizados com estímulos tangíveis sobre a mesa, mesmo que os estímulos possuam grandes dimensões, envolvam a organização de diversos materiais, manuseio complicado e posicionamento em locais bem definidos. Permite-se, o contato físico com os estímulos, adicionando-se as vantagens do uso de recursos informatizados. $\mathrm{O}$ controle experimental rigoroso, o registro fi- 
dedigno de parâmetros (e.g., resposta de escolha, latência, tempo para liberação das consequências e outros) e a mecanização de procedimentos são feitos diretamente por um programa computacional que controla o aparato experimental, e está acoplado a um computador. Esse programa computacional permite a criação de uma vasta biblioteca com os esquemas de reforçamentos, como esquema de reforçamento contínuo, de razão fixa, de razão variável, de intervalo fixo e de intervalo variável; armazena os dados automaticamente e diminui as possibilidades de erros manuais, o que favorece o controle experimental e torna a execução das tarefas de coleta de dados mais rápida e com menor custo de resposta para o pesquisador.

Este aparato pode ser utilizado na avaliação de preferência de tarefas de trabalho, acadêmicas e na avaliação de estímulos de preferência de diferentes categorias sensoriais: estímulos que os indivíduos possam olhar, tocar, sentir, ouvir, experimentar, etc.

A utilização de recursos computacionais parece bastante promissora, com a possibilidade adicional de proporcionar um uso mais frequente e por um maior número de pessoas.

\section{Descrição do Aparato Experimental de Escolha}

O aparato (Figura 1, Painéis A - F) é composto por um tampo de mesa redondo de fibra de média densidade (MDF), na cor branca, medindo 1,20 m de diâmetro e fixado a uma base de metal. Fixado sobre o tampo há uma divisória em MDF, com aproximadamente $50 \mathrm{~cm}$ de altura (a partir do tampo), que divide a mesa em três partes iguais. Também sobre o tampo, em uma das duas partes iguais, encontra-se afixado um dispositivo eletroeletrônico digitalizado medindo $28 \mathrm{~cm}$ x $14 \mathrm{~cm}$ x $2,5 \mathrm{~cm}$ (comprimento x largura $\mathrm{x}$ altura), contendo dois botões interruptores em acrílico translúcido, com diâmetro de 3,5 $\mathrm{cm}$, distantes $10 \mathrm{~cm}$ entre si. Sob cada um desses botões encontram-se cinco diodos emissores de luz ("leds") nas cores: azul, vermelha, verde, branca e amarela. Os botões estão ligados a um contador temporal programável e a outro, de res- postas, por meio de um programa de computador. $\mathrm{O}$ contador registra o número de respostas do participante em cada botão, o tempo gasto em cada um deles, disponibiliza a consequência e o número de consequências obtidas assim que $o$ participante completa o esquema de reforçamento. Sob o tampo há um redutor do tipo cremalheira, entre o motor e o aparato, para dar maior torque ao motor, sobre a qual o tampo gira e um motor elétrico, de 12 volts $(\mathrm{V}), 240$ rotações por minuto (RPM) e 40 Watz (W) de potência, que faz girar a cremalheira.

Procedimentos que empregam esquemas concorrentes têm sido os mais comumente utilizados pela literatura analítico-comportamental para investigar o comportamento de escolha (Pace et al., 1985). O programa computacional "Monitor Aparato" permite a avaliação da preferência de potenciais estímulos reforçadores, tanto sob esquemas concorrentes simples, quanto sob esquemas concorrentes com encadeamento, possibilitando a programação de diversos esquemas de reforçamento.

Esquemas concorrentes foram definidos inicialmente por Ferster e Skinner (1957, p. 724) e envolvem dois ou mais esquemas arranjados independentemente, mas que operam ao mesmo tempo; os reforçadores são produzidos por ambos os esquemas. Esquemas concorrentes com encadeamento envolvem a apresentação simultânea de duas ou mais condições de estímulo, associadas cada uma delas a um esquema específico de reforçamento concorrentemente disponíveis. Cada uma dessas condições é chamada de elo inicial, analogia aos elementos de uma corrente ou cadeia. Uma vez que o indivíduo atende ao esquema de um elo inicial, uma outra condição é apresentada como consequência, referida como elo terminal. Enquanto o indivíduo responde sob o esquema de um elo inicial, o outro continua presente na situação, podendo o indivíduo responder sob o esquema associado a este outro elo. Quando o esquema em vigor no elo terminal for concluído, a consequência programada é apresentada. Esse procedimento separa a eficácia reforçadora do elo terminal das contingências que mantêm o responder neste elo (Catania, 1999). 
Tanto nos esquemas concorrentes, como nos esquemas concorrentes com encadeamento de reforçamento, o experimentador pode escolher um esquema de reforçamento independente, igual ou diferente, e o valor associado ao esquema para cada estímulo. Os esquemas de reforçamento disponíveis são de razão fixa ou variável ou intervalo fixo ou variável. Optou-se

A.

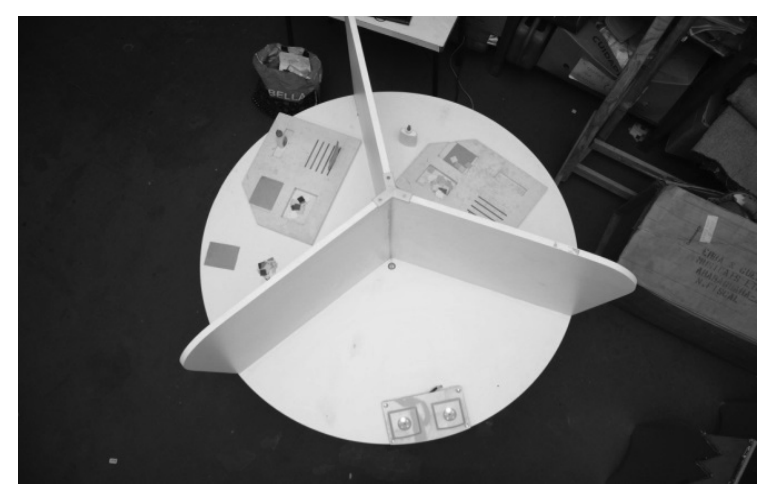

C.

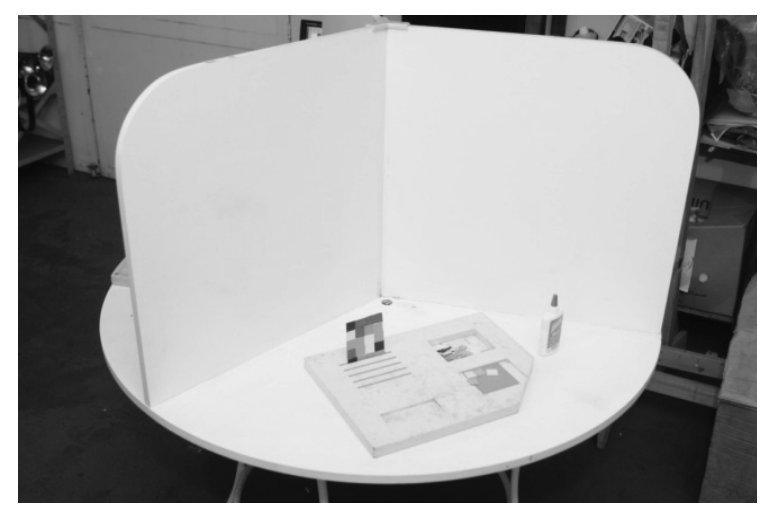

E.

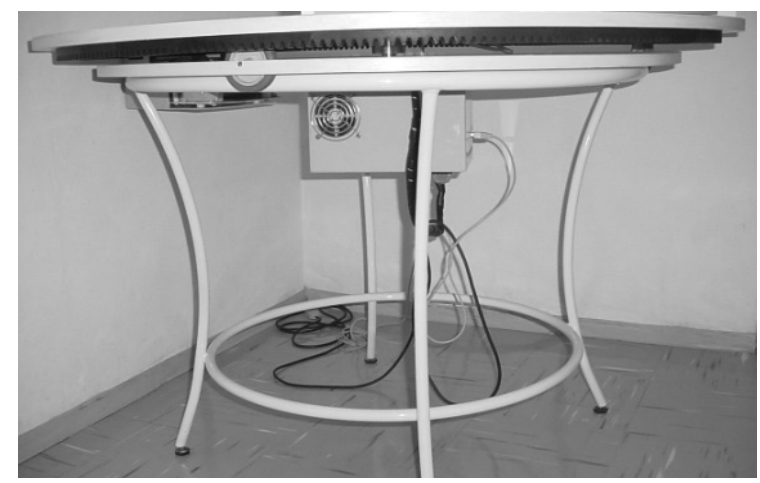

por incluir esquemas de reforçamento de razão fixa, razão variável, intervalo fixo e intervalo variável no programa computacional "Monitor Aparato", pois estudos na área de escolha têm frequentemente se utilizado de diferentes esquemas de reforçamento, a depender dos objetivos e da população utilizada. Alguns estudos mostram, por exemplo, que esquemas fixos

B.

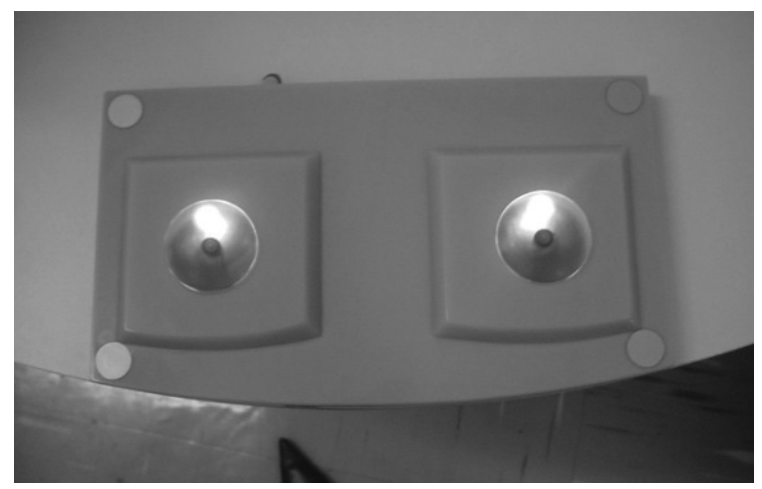

D.

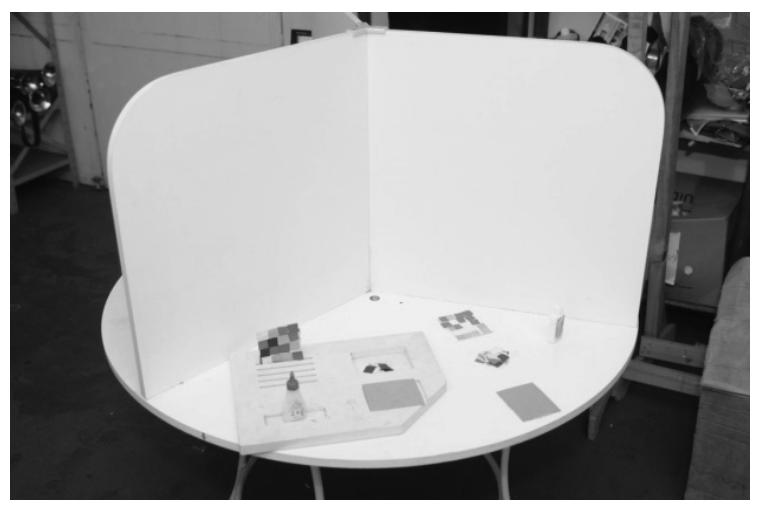

F.

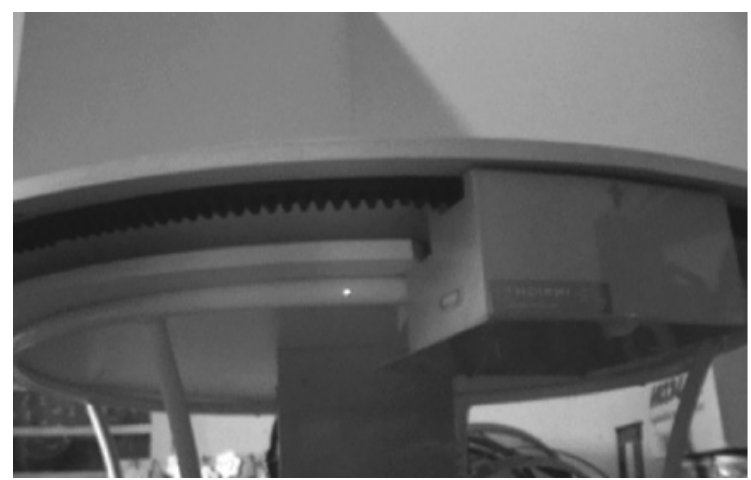

Figura 1. Aparato experimental de escolha (Painéis A - F). O Painel A mostra tomada geral superior do aparato. O Painel B mostra o dispositivo com botões acoplados em detalhe. Os Painéis C e D mostram a divisória com as três partes divididas igualmente. O Painel E mostra uma das partes do aparato onde era disponibilizada uma alternativa de trabalho (condição sem escolha). O Painel F mostra uma das partes do aparato onde eram disponibilizadas duas alternativas de trabalho (condição com escolha). 
produzirem um responder exclusivo em uma das alternativas, com frequência, e por isto, muitos autores optam por esquemas variáveis. Porém, outros estudos mostram que pessoas com deficiência intelectual ou atraso no desenvolvimento têm mais dificuldade de discriminar esquemas variáveis (Pierce \& Cheney, 2004).

O uso de um aparato experimental para estudar escolha e preferência e realizar avaliações de preferência com itens digitais (figuras, sons, vídeos, textos), tanto sob esquemas concorrentes, como sob esquemas concorrentes com encadeamento pode substituir procedimentos que disponibilizam os itens tangíveis sobre a mesa e que são muitas vezes difíceis de serem realizados com muitas vantagens, pois demandam menos esforços do experimentador e frequentemente menos assistentes; menos tempo e organização para apresentação dos estímulos, pois esses procedimentos são mecanizados; e alguns estímulos que não podem ser utilizados (e.g., trem, montanha russa, etc) na avaliação de preferência com estímulos tangíveis podem ser utilizados nesse tipo de avaliação de preferência informatizada.. Adicionalmente, uma ampla gama de estímulos pode fazer parte do banco de dados Portanto, procedimentos realizados no Aparato Experimental de Escolha para avaliar preferências podem representar uma tecnologia promissora de aplicação em ambientes educacionais e clínicos por serem confiáveis e de fácil aplicação, possibilitando economia de tempo e uso mais frequente.

O programa computacional pode ser instalado e executado em qualquer computador compatível com o sistema operacional "Windows", juntamente com o banco de dados Interbase.

O Banco de dados Interbase é responsável pelo armazenamento de informações registradas durante o experimento, e que poderão ser utilizadas posteriormente pelo pesquisador na elaboração de planilhas, relatórios, gráficos, estatísticas, etc. Portanto, é necessária a instalação do Banco de Dados Interbase antes de utilizar o Programa computacional "Monitor Aparato".

A comunicação do Aparato Experimental de Escolha com o computador é feita através de uma porta de comunicação USB 2.0. O equipamento utiliza-se da tecnologia Usb Hid. Essa tecnologia é totalmente "plug" "and" "play".

Após instalar o programa computacional "Monitor aparato", e o banco de dados "Interbase", inserindo-se o CD de instalação e clicando-se duas vezes nos ícones "Monitor aparato" e "Interbase", seguido de um clique em "executar", um ícone com o nome "Aparato" aparecerá na área de trabalho do computador (Figura 2).

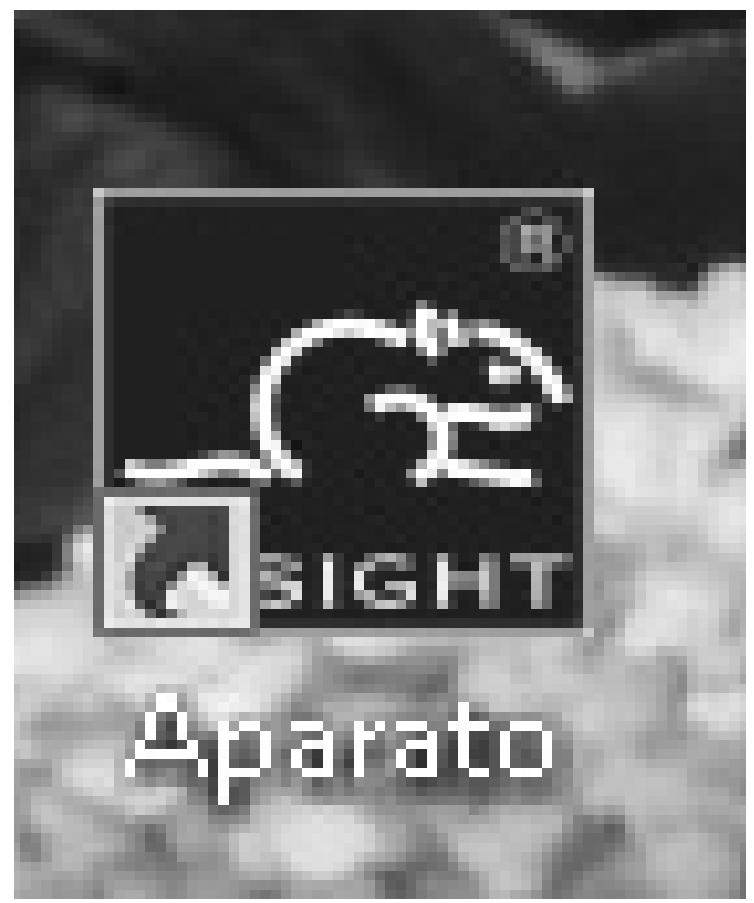

Figura 2. Ícone do aparato experimental de escolha que aparece na área de trabalho após instalar o programa computacional Monitor Aparato.

Ao clicar duas vezes sobre esse ícone, o usuário terá acesso à tela inicial do Aparato Experimental de Escolha "Monitor Aparato" (Figura 3, Painel A). Nesta tela, quatro campos são encontrados na parte superior esquerda, sentido horizontal: "Arquivo", "editar", "ferramentas" e "sobre". Dentro do campo "arquivo", quatro campos são encontrados: "novo", "abrir", "relatórios", "sair" (Figura 3, Painel B). O usuário poderá desenvolver um novo procedimento, clicando no campo "novo", e depois no campo "procedimento". No campo "abrir", o usuário poderá buscar relatórios anteriores presentes no computador e abrir dentro do programa computacional "Monitor aparato". No campo "relatórios", o usuário poderá buscar e acessar relatórios anteriores. No campo "sair", o usuário sairá do programa computacional "Monitor aparato". 
No campo "editar", ao lado do campo arquivo, horizontalmente, dois campos serão encontrados: "participantes" e "campo" (Figura 3, Painel C). O usuário poderá editar informações de participantes (Figura 3, Painel D) e de campos (local em que se encontram os campos: "tentativa", "inatividade", "fim da tentativa", "reforço", "sessão", "reforço botão direito", "reforço botão esquerdo", "intervalo", "intervalo médio", "desvio padrão médio", "duração", "horário", "anti-horário", "cor led direito", "cor led esquerdo", "blocos de incremento", "valor médio das razões", "desvio médio", "razão", "quantidade respostas", "razão inicial", "intervalo inicial", "esquemas de reforços", "valores"), presentes no programa computacional "Monitor aparato" (Figura 3, Painel E). No campo "ferramentas", ao lado do campo "editar", horizontalmente, o usuário poderá fazer um teste do equipamento (Figura 3, Painel F). No campo "sobre", tanto no campo "versão", como no campo " manual do usuário", estão presentes informações gerais e de instalação do programa computacional e informações de uso do Aparato Experimental de Escolha (Figura 3, Painéis G e H).

Para programar uma tarefa o passo inicial é selecionar em "arquivo", o campo "novo", e depois selecionar o campo "procedimento" na tela "Monitor aparato" (Figura 3, Painel B). Em seguida, aparece a tela de programação da tarefa "Editor de procedimento" (Figura 3, Painel $\mathrm{K})$. O experimentador clica no campo "Botão direito", na parte superior esquerda dessa tela, escolhe um esquema de reforçamento para o botão direito, na parte inferior esquerda dessa tela, clicando uma vez em um dos esquemas de reforço que aparecem verticalmente. Ao clicar no campo "Apresentação de reforços", na parte superior direita dessa tela, três novos campos são abertos: "identificador", "esquema de reforços" e "razão" (Figura 3, Painel J). O experimentador pode escolher um esquema de reforçamento independente, igual ou diferente, e o valor associado ao esquema para cada botão. No campo "identificador", o usuário deverá colocar o valor do esquema de reforçamento de razão (fixo ou variável) ou de intervalo (fixo ou variável) digitando o número desejado no campo que se abre em branco. No campo "esquema de reforços", aparecerá automaticamente o tipo de esquema escolhido e no campo "razão", o mesmo valor colocado no campo "identificador" deverá ser colocado. Ao clicar no campo "Configuração de tempo", na parte superior direita dessa tela, ao clicar, abaixo do campo "Apresentação de reforços", cinco campos são abertos: "tentativa", "inatividade", "reforço", "fim tentativa" e "sessão" (Figura 3, Painel K).

Os campos "tentativa", "inatividade" "fim tentativa" e "sessão", devem ser preenchidos quando, respectivamente, se quer especificar um tempo total para a realização de uma tentativa; se quer especificar um tempo para o tampo do Aparato Experimental de Escolha retornar à posição inicial, após um determinado tempo em que haja ausência de respostas; se quer especificar um tempo total que não deva ser ultrapassado para cada tentativa ou sessão. No campo "reforço",tem-se a possibilidade de colocar quanto tempo deverá passar até que a consequência seja disponibilizada. O tempo poder ser colocado em horas, minutos ou segundos. Após completar o esquema de reforçamento, o tampo do Aparato Experimental de Escolha gira, na direção horário ou anti-horário, dependendo das configurações para cada botão, e disponibiliza a consequência pelo tempo pré-determinado pelo experimentador. Após passar esse tempo, o tampo do Aparato Experimental de Escolha retorna para a posição inicial (posição com a presença dos botões) ou o experimentador pode clicar em qualquer área branca da tela para retornar para a posição inicial. Após passado o tempo pré-determinado, o tampo do aparato gira na posição inicial com botões.

Ao clicar no campo "Propriedades", na parte superior direita dessa tela, abaixo do campo "Configuração de tempo", ao lado dos campos "botão esquerdo" e "botão direito", aparecerá um "menu" de opções de cores para serem selecionadas para cada botão e de rotação selecionada para cada botão (Figura 2, Painel L). Para acessar o "menu" de cores, basta clicar com o botão direito do "mouse" no lado desejado (Figura 3, Painel M). Abaixo do "menu" de cores, um "menu" de rotação do tampo (sentido horário e anti-horário) do Aparato Experimental de Escolha associado a cada botão estará presen- 
te (Figura 3, Painel N). Um clique sobre a rotação desejada seleciona a rotação do tampo. Em seguida, no campo "critério de finalização da sessão", na parte inferior direita dessa tela, o experimentador deve colocar nos quadrados em branco ao lado esquerdo dos parâmetros acertos, erros ou tentativas, um número que indique após quantos acertos, erros ou tentativas, a sessão deverá ser encerrada (Figura 3, Painel L, parte inferior direita). Os parâmetros erros, acertos ou tentativas são escolhidos arbitrariamente pelo experimentador, conforme suas necessidades de pesquisa. Após realizar esses passos, o experimentador clica em "aplicar", na parte inferior direita dessa tela. Realiza procedimento análogo para o botão esquerdo. Após isto, aparece a tela de informação da sessão "Editor sessão" em que o experimentador preenche seu nome no campo "pesquisador" e fornece um título à sessão no

Painel A

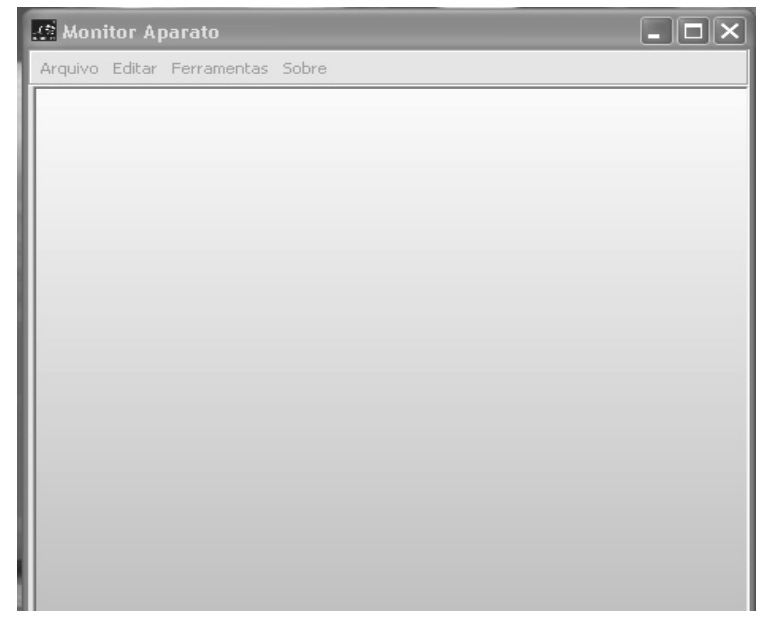

Painel C

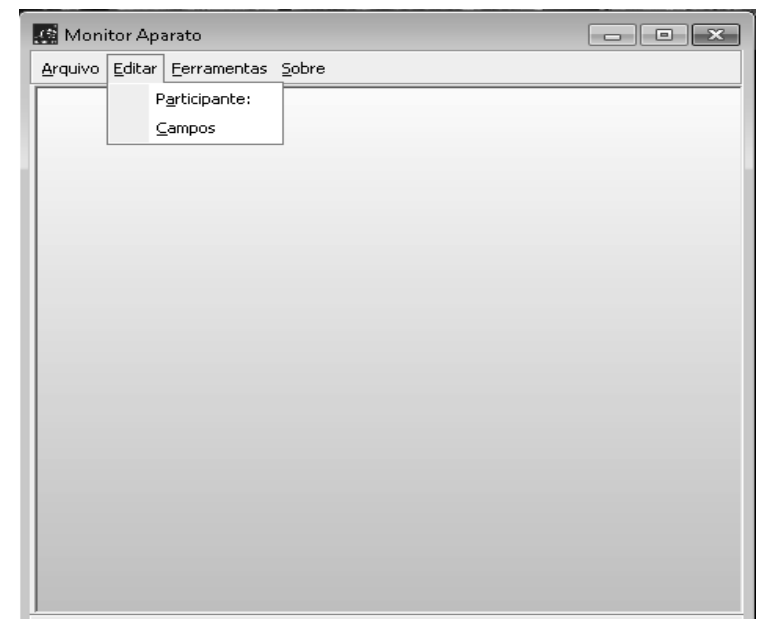

campo "título" (Figura 3, Painel O). Após configuradas as informações, clica-se uma vez no campo "salvar". Aparece, então, a tela de informações do participante "Pesquisa de participante no banco de dados" (Figura 3, Painel P). Se o participante não tiver cadastro, o experimentador realiza o cadastro na parte direita dessa tela com informações, tais como: nome, idade, sexo, endereço, bairro, cidade, CEP, UF e telefone e salva o cadastro na tecla "salvar", na parte inferior direita dessa tela, que aparece após a realização do cadastro. Se o participante já tiver sido cadastrado, o experimentador clica na primeira tecla à esquerda na parte inferior dessa tela e os participantes cadastrados aparecem listados na parte superior esquerda dessa tela. Em seguida, o experimentador clica sobre o nome selecionando e clica na quarta tecla, na parte inferior esquerda dessa tela, para iniciar a sessão localizada.

Painel B

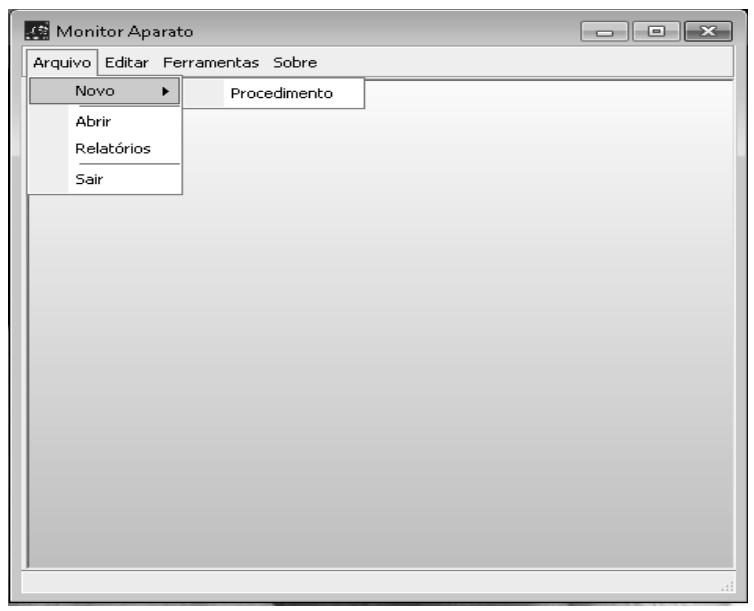

Painel D

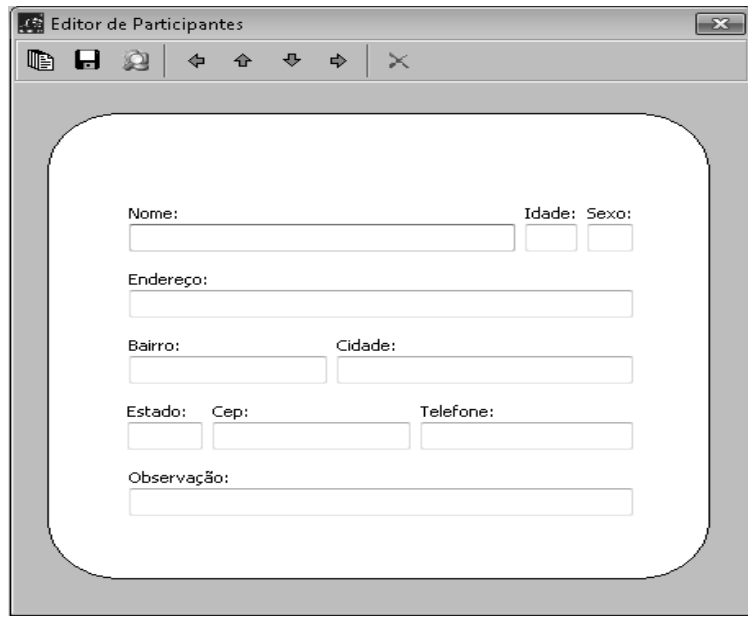


Painel E

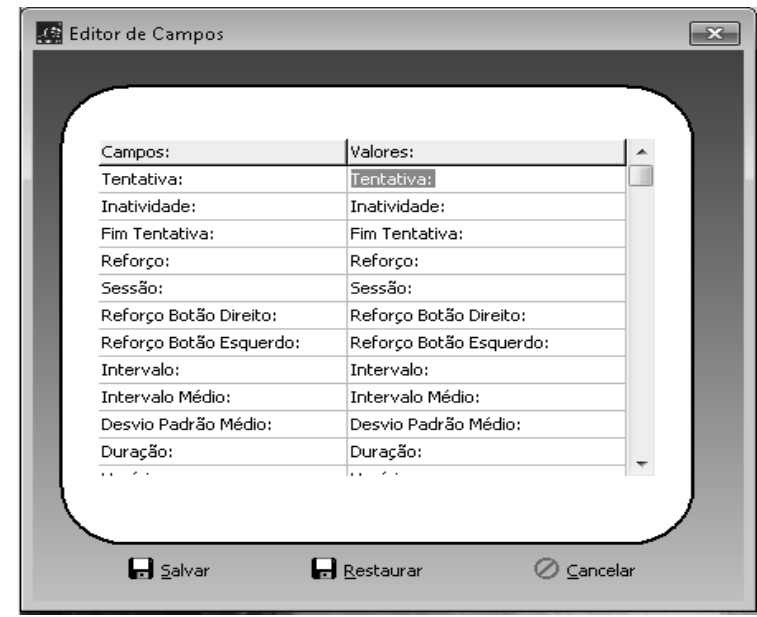

Painel G

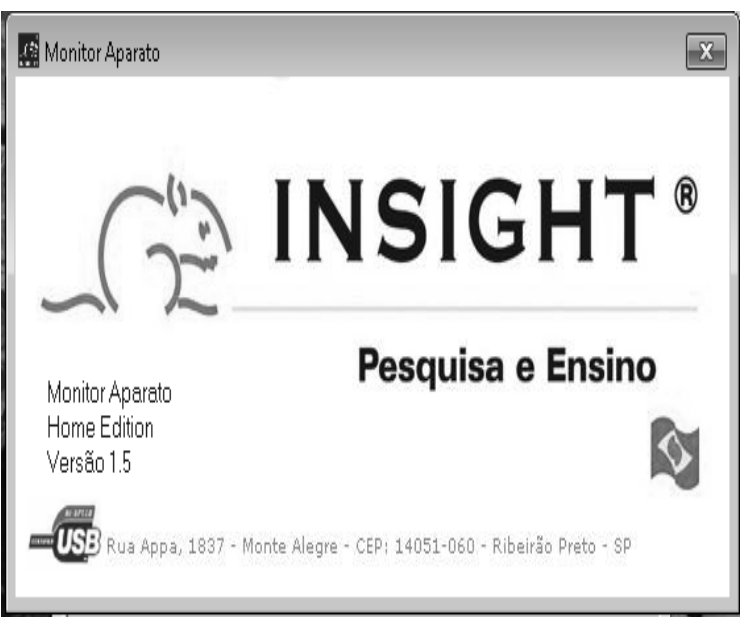

Painel I

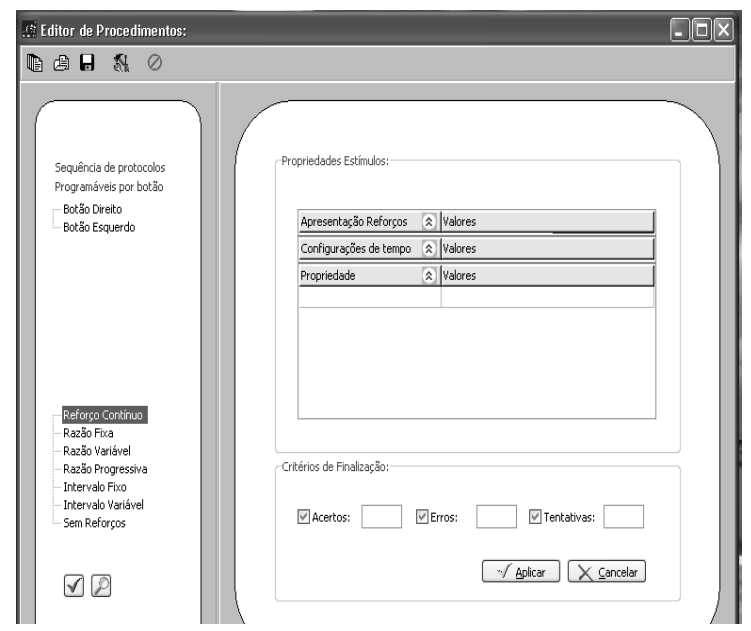

Painel F

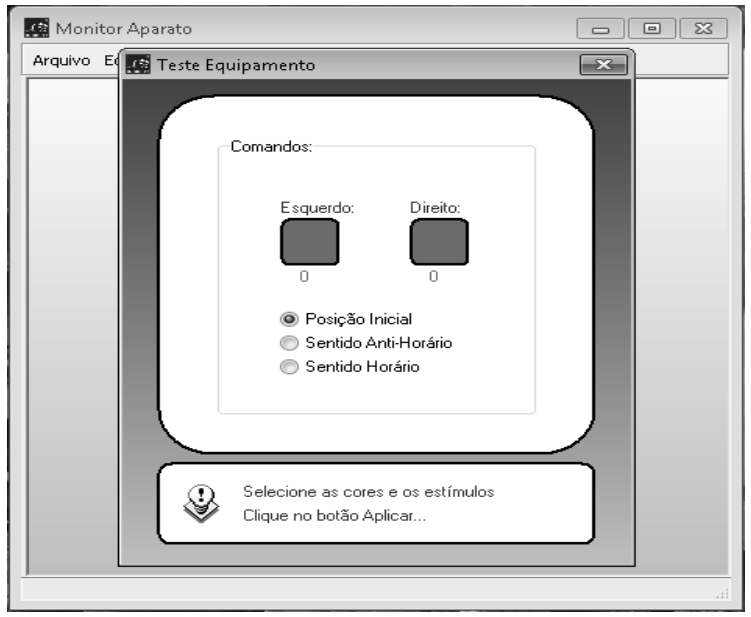

Painel $\mathrm{H}$

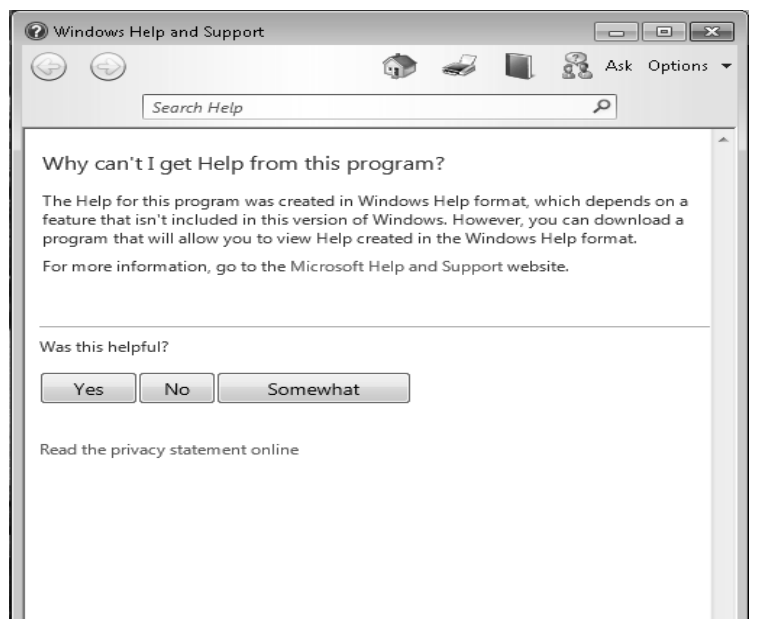

Painel J

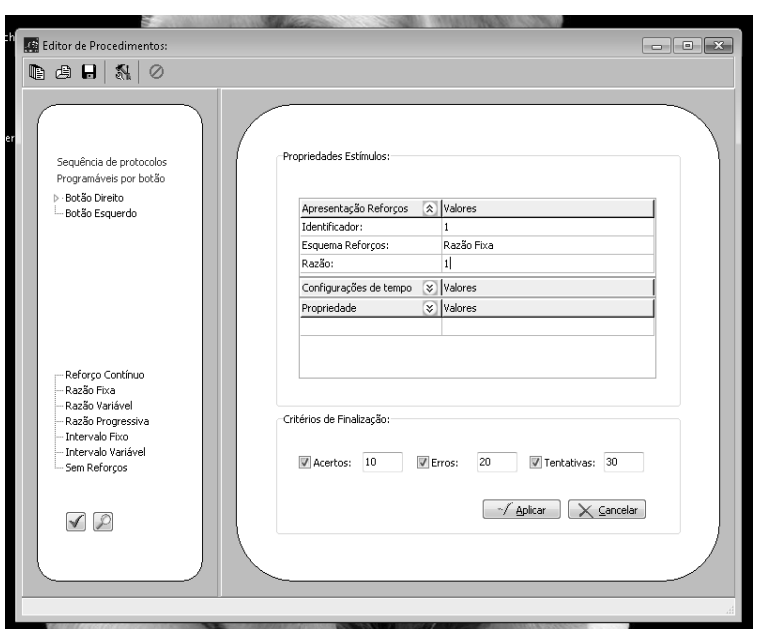


Painel K

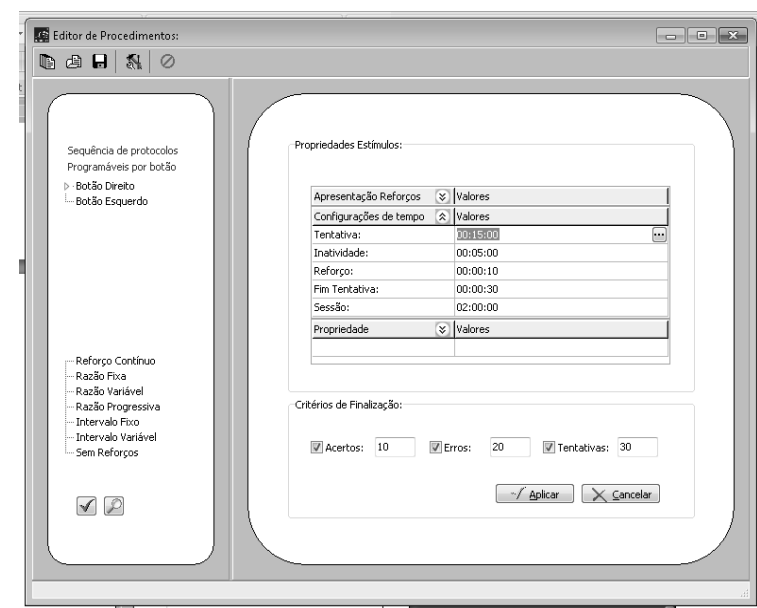

Painel M

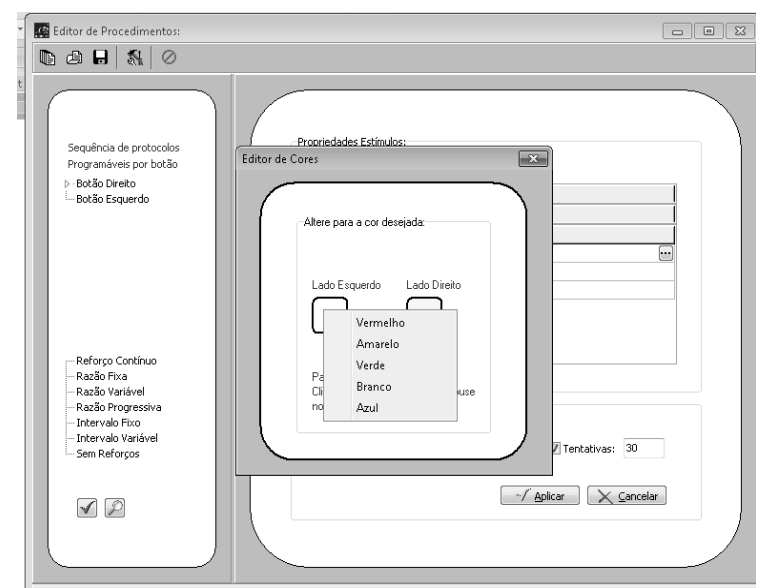

Painel O

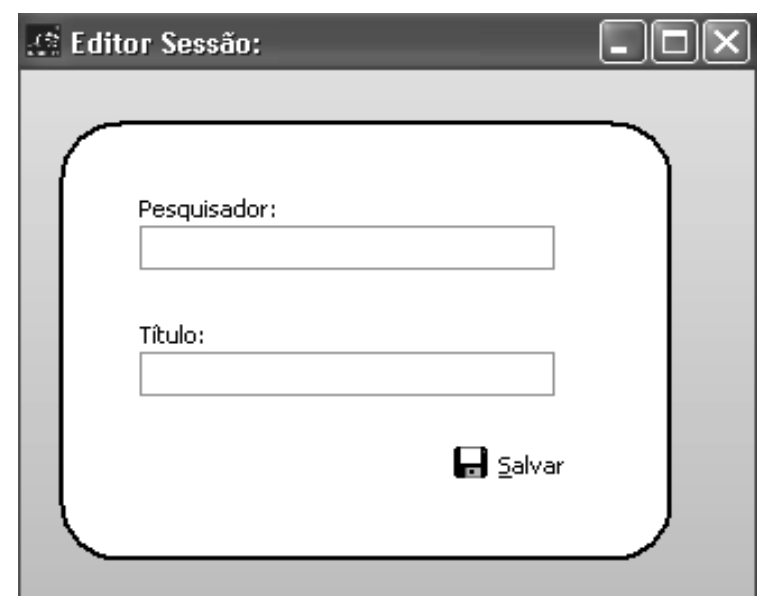

Painel L

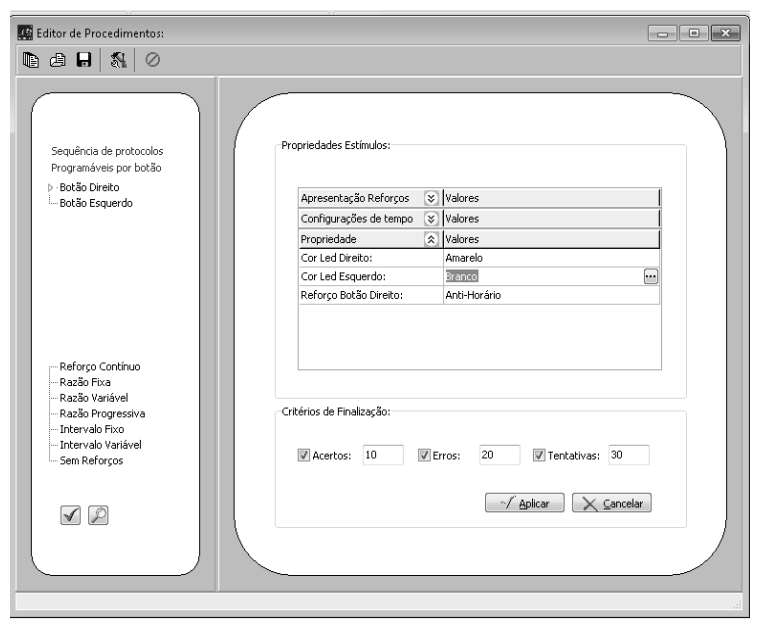

Painel N

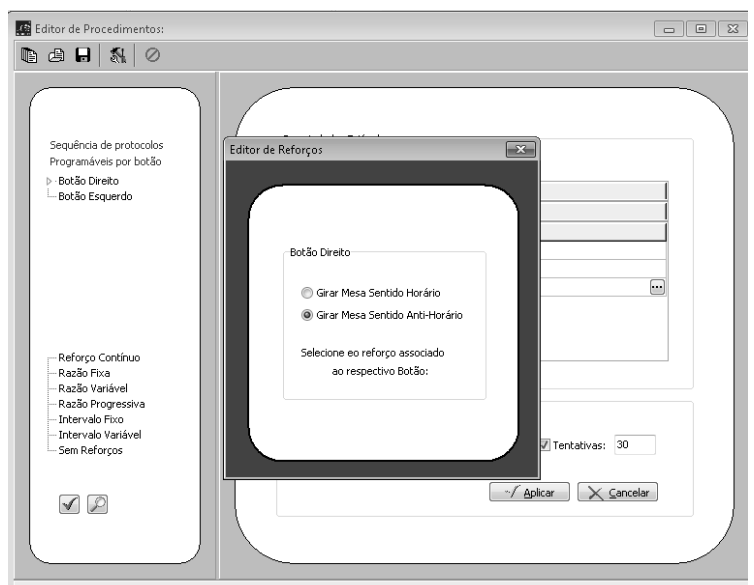

Painel $\mathrm{P}$

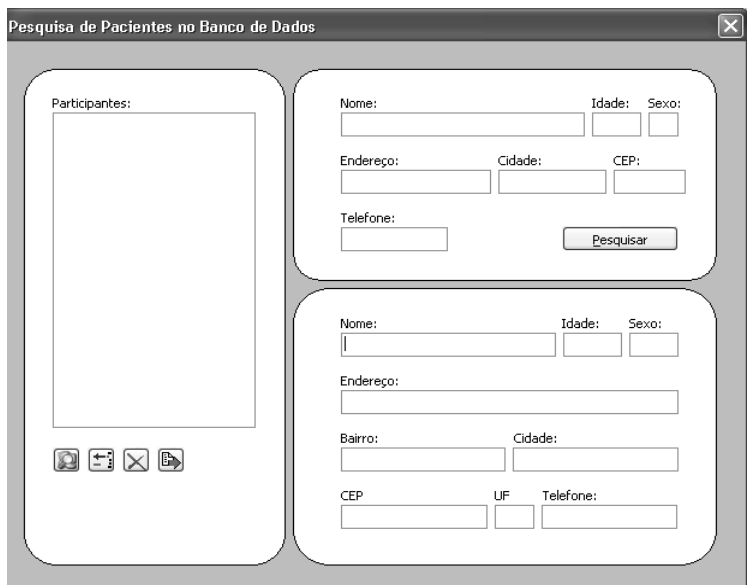

Figura 3. (Painéis A, B, C, D. E, F, G, H, I, J, K, L, M, N, O e P). Telas de configuração da sessão.

Nos relatórios emitidos pelo programa computacional do Aparato Experimental de Escolha, são consideradas as escolhas para os estímulos apresentados simultaneamente na tela do computador ou nos botões do Aparato Experimental de Escolha para escolha. Nesse relatório, registra-se o nome do experimentador, o nome do participante, a data, o número da tentativa, $o$ 
horário de início e de término da tarefa, o estímulo que estava na posição esquerda, o estímulo que estava na posição direita, a frequência, a frequência acumulada, a resposta correta, a resposta emitida pelo participante, se foi ou não apresentada consequência para a resposta emitida, e em quantos segundos a resposta foi emitida (latência; Figura 4).

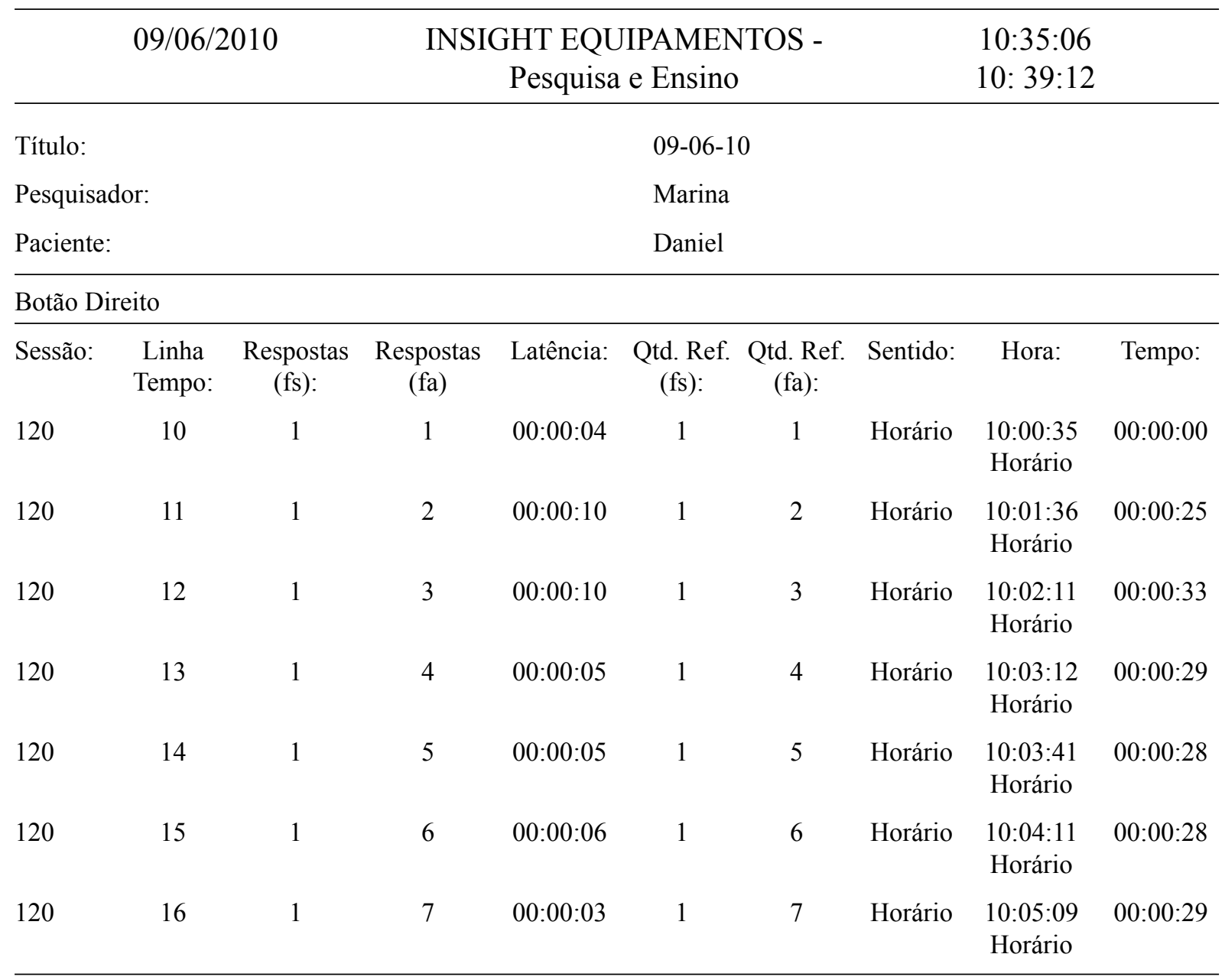

Botão Esquerdo

\begin{tabular}{|c|c|c|c|c|c|c|c|c|c|}
\hline Sessão: & $\begin{array}{l}\text { Linha } \\
\text { Tempo: }\end{array}$ & $\begin{array}{l}\text { Respostas } \\
\text { (fs): }\end{array}$ & $\begin{array}{l}\text { Respostas } \\
\quad \text { (fa) }\end{array}$ & Latência: & $\begin{array}{l}\text { Qtd. Ref. } \\
\text { (fs): }\end{array}$ & $\begin{array}{l}\text { Qtd. Ref. } \\
\text { (fa): }\end{array}$ & Sentido: & Hora: & Tempo: \\
\hline 120 & 9 & 1 & 1 & 00:00:23 & 1 & 1 & Horário & $\begin{array}{l}\text { 10:00:07 } \\
\text { Horário }\end{array}$ & 00:00:00 \\
\hline 120 & 10 & 1 & 2 & 00:00:02 & 1 & 2 & Horário & $\begin{array}{l}\text { 10:01:02 } \\
\text { Horário }\end{array}$ & 00:00:26 \\
\hline 120 & 11 & 1 & 3 & 00:00:07 & 1 & 3 & Horário & $\begin{array}{l}\text { 10:02:42 } \\
\text { Horário }\end{array}$ & 00:00:34 \\
\hline 120 & 12 & 1 & 4 & 00:00:05 & 1 & 4 & Horário & $\begin{array}{l}\text { 10:04:41 } \\
\text { Horário }\end{array}$ & 00:00:28 \\
\hline 120 & 13 & 1 & 5 & 00:00:02 & 1 & 5 & Horário & $\begin{array}{l}\text { 10:05:35 } \\
\text { Horário }\end{array}$ & $00: 00: 26$ \\
\hline
\end{tabular}


O sistema informatizado "Aparato de escolha" disponibiliza, portanto, uma possibilidade de tarefa estruturada para estudo do comportamento de escolha, de acordo com a literatura em Análise Experimental do Comportamento, para tornar mais acurado o estudo desse comportamento e permitir a realização de avaliações de preferência com estímulos de difícil manipulação e que poderiam ser excluídos dessas avaliações. Além de possibilitar registros mais fidedignos do comportamento de escolha, a apresentação das e diminuição do custo de resposta por parte do pesquisador, pois o experimentador programa a sessão apenas uma vez para cada formato de sessão, e depois o mesmo simplesmente seleciona a sessão programada e o participante ao realizar uma nova sessão, sendo essa uma vantagem do aparato.

O Aparato Experimental de Escolha para avaliação de preferência por tarefas de trabalho, estímulos de preferência e condições sem e com escolha apresenta uma tecnologia promissora de aplicação em diversos ambientes, é mais fidedigna no registro, possibilitando economia de tempo e uso mais frequente por mais profissionais. O sistema informatizado "Aparato de escolha" destina-se a profissionais que atuam na área de educação em geral, educação especial, psicologia e áreas afins. A maior limitação do Aparato Experimental de Escolha, refere-se a sua portabilidade. Ele é grande, pesado e necessita de cuidados especiais para ser transportado. Portanto, para uso em um ambiente fixo, ele é extremamente útil, porém, para utilizá-lo em diversos ambientes, seu uso fica restrito e bastante dificultado. Adicionalmente, seu custo é elevado e seu manuseio é de relativa complexidade e requer treinamento, e algum conhecimento da literatura de escolha e esquemas de reforçamento é também necessário.

\section{Referências}

Carr, J. E., Nicolson, A. C., \& Higbee, T. S. (2000). Evaluation of a brief multiple-stimulus preference assessment in a naturalistic context. Journal of Applied Behavior Analysis, 33, 353-357.
Catania, A. C. (1999). Aprendizagem: Comportamento, linguagem e cognição (4. ed., D. G. de Souza, Trad.). Porto Alegre, RS: Artes Médicas Sul.

Conyers, C., Doole, A.,Vause, T., Harapiak, H., Yu, D. C. T., \& Martin, G. L. (2002). Predicting the relative efficacy of three presentation methods for assessing preferences of persons with developmental disabilities. Journal of Applied Behavior Analysis, 35, 49-58.

DeLeon, I. G., \& Iwata, B. A. (1996). Evaluation of a multiple-stimulus presentation format for assessing reinforcer preferences. Journal of Applied Behavior Analysis, 29, 519-533.

Dunlap, G., DePerczel, M., Clarke, S., Wilson, D., Wright, S., White, R., \& Gomez, A. (1994). Choice making to promote adaptative behavior for students with emotional and behavioral challenges. Journal of Applied Behavior Analysis, 27, 505-518.

Escobal, G., \& Goyos, A. C. N. (2008). Análise das variáveis determinantes do comportamento de escolha entre alternativas de trabalho em adultos com deficiência mental. Revista Brasileira de Análise do Comportamento, 4, 71-87.

Ferster, C. B., \& Skinner, B. F. (1957). Schedules of reinforcement. New York: Appleton-Century-Crofts.

Fisher, W. W., Piazza, C. C., Bowman, L. G., Hagopian, L. P., Owens, J. C., \& Slevin, I. (1992). A comparison of two approaches for identifying reinforcers for persons with severe and profound disabilities. Journal of Applied Behavior Analysis, 25, 491-498.

Fisher, W. W., Thompson, R. H., Piazza, C. C., Crosland, K. A., \& Gotjen, D. (1997). On the relative reinforcing effects of choice and differential consequences. Journal of Applied Behavior Analysis, 30, 423-438.

Groskreutz, M. P., \& Graff, R. B. (2009). Evaluating pictorial preference assessment: The effect of differential outcomes on preference assessment results. Research in Autism Spectrum Disorders, 3, 113-128.

Hanley, G. P., Iwata, B. A., \& Lindberg, J. S. (1999). Analysis of activity preferences as a function of differential consequences. Journal of Applied Behavior Analysis, 32, 419-435.

Hanna, E. S., \& Ribeiro, M. R. (2005). Autocontrole: Um caso especial de escolha. In J. Abreu-Rodri- 
gues \& M. R. Ribeiro (Eds.), Análise do comportamento: Pesquisa, teoria e aplicação (pp. 175-187). Porto Alegre, RS: Artmed.

Higbee, T. S., Carr, J. E., \& Harrison, C. D. (1999). The effects of pictorial versus tangible stimuli in stimulus-preference assessments. Research in Developmental Disabilities, 20(1), 63-72.

Luckasson, R., Borthwick-Duffy, S., Buntinx, W. H. E., Coulter, D. L., Craig, E. M., Reeve, A., ... Tassé, M. J. (2002). Mental retardation - Definition, classification, and systems of supports (10 ${ }^{\text {th }}$ ed.) Washington, DC: American Association on Mental Retardation.

Luckasson, R., Coulter, D. L., Polloway, E. A., Reiss, S., Scalock, R. I., Snell, M. E., ...Stark, J. A. (1992). Mental retardation - Definition, classification, and systems of supports $\left(9^{\text {th }}\right.$ ed.) Washington, DC: American Association on Mental Retardation.

Pace, G. M., Ivancic, M. T., Edwards, G. L., Iwata, B. A., \& Page, T. J. (1985). Assessment of stimulus preference and reinforcer value with profoundly retarded individuals. Journal of Applied Behavior Analysis, 18, 249-255.
Piazza, C. C., Fisher, W. W., Bowman, L. G., \& Blakeley-Smith, A. (1999). Identifying and assessing reinforcers using choice paradigms. In $\mathrm{P}$. M Ghezzi, W. L. Williams, \& J. E. Carr (Eds.), Autism: Behavior analytic perspectives (pp. 101-107). Reno, NV: Context Press.

Pierce, W. D., \& Cheney, C. D. (2004). Behavior analysis and learning. Mahwah, NJ: Lawrence Erlbaum.

Roane, H. S., Vollmer, T. R., Ringdahl, J. E., \& Marcus, B. A. (1998). Evaluation of a brief stimulus preference assessment. Journal of Applied Behavior Analysis, 31, 605-620.
Recebido: 14/06/2013

$1^{a}$ revisão: $30 / 10 / 2013$

$2^{a}$ revisão: 02/12/2013

Aceite final: 08/01/2014 